\title{
Investments, positive externalities and majority bargaining
}

Daniel Cardona

Universitat de les Illes Balears

E-mail: d.cardona@uib.cat

Antoni Rubí-Barceló Universitat de les Illes Balears

E-mail: antoni.rubi@uib.eu

May 2017 


\title{
Investments, positive externalities and majority bargaining
}

by

\author{
Daniel Cardona and Antoni Rubí-Barceló*
}

\begin{abstract}
May 19, 2017
This paper analyzes the welfare implications of requiring either unanimity or simple majority in negotiations to distribute a budget among agents who previously can invest to generate positive consumption externalities to others. The present paper studies this setting with simple-majority bargaining, complementing Cardona and Rubí-Barceló (2014a), that consider the unanimity case. It is shown that reducing the majority requirement reduces the profitability of investments and, as a consequence, alleviates over-investment, which is predominant under unanimous bargaining. Nevertheless, simple majority reduces the aggregate surplus attained at the bargaining stage. Therefore, the relative performance of the bargaining rules is uncertain. We show how it evolves with respect to the size of consumption externalities.
\end{abstract}

(JEL: C78, D72)

\section{Introduction}

In a variety of situations, the members of a committee may invest to 'open the way' to spreading positive externalities to the others before engaging in a multilateral bargaining game to distribute a divisible good among them. These situations range from $(i)$ productivity investments by the branch-managers of a firm preceding the negotiations to distribute a given budget among them to (ii) legislative budgeting environments in which legislators may previously 'shape' the projects they will propose to be voted. For example, before negotiating how to split a given budget among legislators' projects, each of them may invest to increase the benefits of her project for all others. Cardona and Rubí-Barceló (2014a) analyze the theoretical framework fitting these and other examples, under the particularity that unanimous consent is required to approve any proposed distribution of the budget. The present paper extends this analysis to simple-majority consent and study the consequences on efficiency of this alternative voting rule.

The following setting is considered: A committee has a budget to be divided among their members with the particularity that individual shares may generate consumption externalities to all agents (including themselves) or not, depending on whether they face a previous investment. After taking investment decisions, agents negotiate the distribution of this budget following a standard legislative bargaining process, à la Baron and

*Departament d'Economia Aplicada, Universitat de les Illes Balears. We acknowledge financial support from the Generalitat de Catalunya through grant SGR2009-1051 and Ministerio de Ciencia y Tecnologia through grant ECO2009-06953. 
Ferejohn (1989), where agreements require the acceptance of at least half of the agents. ${ }^{1}$ The existence of positive consumption externalities would lead to under-investment at the initial stage, as in a classical public-good game. However, investments provide a more favorable bargaining position to investing agents because others are more willing to give them a bigger share in the ensuing negotiation. This causes a rent-seeking behavior that may lead agents' investments to indirectly 'hurt' others. This would cause over-investment in equilibrium, as agents do not consider this negative external effect when deciding. As a result of the interaction between these two opposite forces, Cardona and Rubí-Barceló (2014a) show that, under unanimity, equilibria can be efficient but they may also display either under- or over-investment. Moreover, since this consensus requirement is particularly profitable for investing agents (because they must be included in any agreement), the inefficiency pattern that predominates in that setting is over-investment. Reducing the consensus requirement to simple majority has two opposite effects on efficiency: First, some of the benefits from investing are eroded because those who invested can now be excluded from the winning coalition. This lower profitability mitigates over-investment. Second, excluding investing agents from the winning coalition may imply a reduction of the budget received by them in the bargaining equilibrium. Due to the non-transferability of utilities, this means that the collective surplus would not be maximized. ${ }^{2}$ This paper intends to illustrate the balance between these two opposite effects. Our results show that simple majority generates more surplus than unanimity when consumption externalities are sufficiently large. Otherwise, the rent dissipation caused by the allocation of some budget to non-investing agents might exceed the positive effects of reducing over-investment at the initial stage, so outcomes under unanimity are more efficient than under simple majority.

Our study analyzes how investment decisions and redistribution depend on the specificity of the decision process; in particular, the required consensus at the negotiation stage. Thus, this paper contributes to the literature about the performance of alternative majority rules. Additionally, our model analyzes individual investments in collective decisions, which is the main issue in the huge literature addressing the hold-up problem. This literature studies the how the nature of investments (basically, their effects on the ensuing bargaining game) alter the possibilities that the rents generated by individuals are, at least partially, expropriated (as a short sample see e.g., Che and Hausch (1999), de Meza and Lockwood (2010), Grout (1984), Hart and Moore (1988) or Rogerson (1992).) Related to our work is also Harstad (2005), that studies the effects of majority rules on investment decisions in a binary choice collective decision problem. The author models the collective decision process as a proto-coalition majority bargaining game where unanimity is required within the coalition. Investments affect the utility of the investing agent and possibly to others when one of the alternatives is selected. His results show that when unanimity is required, the usual under-investment problem

\footnotetext{
${ }^{1}$ Calvert and Dietz (2005) study a three-player simple-majority bargaining game with symmetric positive consumption externalities.

${ }^{2}$ Note that externalities make utilities non-transferable and that collective surplus is maximized only when the budget is entirely allocated to investing agents.
} 
arises. However, he assumes partial transferability of utilities, in the sense that they are perfectly transferable only among agents within the formed coalitions, but that there is some utility loss when transferring utility from the excluded agents to the coalition members. This implies that investing agents generate more collective surplus when they are included in the winning coalition. Moreover, as any winning coalition may expropriate outsiders, this generates strong incentives to invest, and this effect can induce to over-investment when the majority is low enough. The paper characterizes the efficient (super) majority rule by finding the consensus requirement that balances such incentives with the hold-up problem of under-investment. Hence, while Harstad (2005) shows that simple majority enhances investments, our results suggest the opposite when considering a legislative bargaining game without transferable utilities.

In the next section we present the model. In Section 3, we study the properties of investments when either unanimity or simple majority conduct negotiations. Section 4 performs a welfare analysis of the outcomes obtained under alternative consensus requirements; and Section 5 concludes.

\section{The model}

The model we consider builds on Cardona and Rubí-Barceló (2014a), henceforth CRB. A committee of three players $N=\{1,2,3\}$ follows a two-stage game to distribute a fixed divisible budget among them. First, agents individually decide whether to invest $c>0$, and second they negotiate about how to divide the budget through standard legislative bargaining. Each agent benefits from her own share but also from the share allocated to those agents who invested in the first stage. That is, the shares of investing agents generate positive consumption externalities. Negotiations proceed over discrete infinite time as follows: at each period $t \geq 0$ one player $j \in N$ is randomly selected as the proposer, with equal probability each. This player proposes an allocation of the budget $\mathbf{x}^{j}=\left(x_{1}^{j}, x_{2}^{j}, x_{3}^{j}\right) \in X=\left\{\left(x_{1}, x_{2}, x_{3}\right) \in \mathbb{R}_{+}^{3}: \sum_{i=1}^{3} x_{i} \leq 1\right\}$, where $x_{i}^{j}$ denotes the part of the budget assigned to player $i$. Then, sequentially all other players respond by either accepting the proposal or not. Simple majority is required for approval, so the budget is divided as prescribed by the proposal when it receives the support of at least two players. In case of approval, the proposed allocation is implemented and the game ends; otherwise the game moves to period $t+1$ with a new randomly chosen proposer.

We denote by $m_{i} \in\{0,1\}$ the investment choice of agent $i$, where $m_{i}=1$ when $i$ invests and $m_{i}=0$ otherwise. Let $\mathbf{m} \in M=\left\{\left(m_{1}, m_{2}, m_{3}\right): m_{i} \in\{0,1\}, i=1,2,3\right\}$ denote the investment choice profile, and $m=\sum_{i=1}^{3} m_{i}$ denote the number of investing agents. Let $h$ and $l$ denote a generic investing and non-investing member, respectively.

Agents' preferences are defined over both the particular share of the budget they receive, and the part of the budget assigned to $h$-agents. Specifically, upon agreement on $\mathbf{x} \in X$ at period $t$ player $j \in N$ obtains utility $\delta^{t} u_{j}(\mathbf{x})-c \cdot m_{j}$, where ${ }^{3}$

$$
u_{j}(\mathbf{x})=x_{j}+\beta y,
$$

\footnotetext{
${ }^{3}$ Although the surplus is transferable, utility is not.
} 
where $\beta \in(0,1)$ and $y=\sum_{i \in N} m_{i} x_{i}$. Perpetual disagreement yields utility $-c \cdot m_{j}$.

Previous to negotiations, agents make their investment decisions that shape $\mathbf{m}$. Then, negotiations start. We denote by $G(\mathbf{m})$ the subgame attained when $\mathbf{m}$ is played. We will restrict our analysis to equilibria where agents use stationary strategies defined in the following sense: (i) proposals depend only on $\mathbf{m}$, and (ii) response rules depend only on $\mathbf{m}$ and the proposal itself. Hence, at any $G(\mathbf{m})$, a profile of stationary strategies induces constant expected outcomes $\bar{u}_{1}(\mathbf{m}), \bar{u}_{2}(\mathbf{m}), \bar{u}_{3}(\mathbf{m})$ at any bargaining stage. This would determine the acceptance sets $A_{i}(\mathbf{m})=\left\{\mathbf{x} \in X: u_{i}(\mathbf{x}) \geq \delta \bar{u}_{i}(\mathbf{m})\right\}$, and optimal

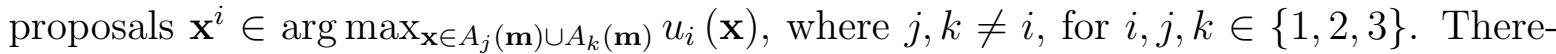
fore, a stationary subgame perfect equilibrium (SSPE) would determine both a partition of the population $\mathbf{m}^{*}$ and a vector of expected utilities $\bar{u}_{j}\left(\mathbf{m}^{*}\right)$ for any $j \in N$ such that: (1) $\bar{u}_{j}\left(\mathbf{m}^{*}\right)$ is the agent $j$ 's expected utility in an SSPE of the multilateral bargaining game $G\left(\mathbf{m}^{*}\right)$; and (2) no player has incentives to change her investment decision. I.e., $m_{i}^{*} \in \arg \max _{m \in\{0,1\}} \bar{u}_{i}\left(m, m_{-i}^{*}\right)-m c{ }^{4}$ We will say that agent $i$ compensates agent $j$ when $\mathbf{x}^{i} \in A_{j}(\mathbf{m})$. Finally, an equilibrium partition is said to be stable.

When $\delta<1$ it is shown (see Appendix A.1) that any SSPE is a no-delay equilibrium. That is, in the equilibrium path any proposal is accepted. Based on this result, to simplify the exposition we will perform our analysis by considering perfectly patient players when agreements require simple majority (i.e., $\delta=1$ ) by studying no-delay SSPE only. Although our results can be derived by considering strictly impatient players, the outcomes we obtain coincide with those obtained when impatience vanishes. Thus, this is without loss of generality. This is not the case in the presence of veto players, as in unanimous bargaining, where impatience is crucial to obtain a unique bargaining outcome. In these cases, the limit outcome is considered.

ASSUMPTION 1 Most of our results would depend on the relationship between $c$ and $\beta$ or between $\beta$ and some real number. For expositional purposes we opt to present our results only generically. That is, some parameters as $\beta \in\{1 / 3,1 / 2,3 / 4\}$ or $c=f(\beta)$ for some function $f$ are generically excluded from the analysis throughout.

\section{Stability versus Efficiency}

In this section, we characterize the SSPE of the strategic game defined in the previous section and determine its efficiency properties. We will say that a partition $\mathbf{m}$ is $r$ efficient if the surplus that generates at the bargaining stage is maximized. That is,

$$
W_{\mathbf{m}}=\sum_{j \in N} \bar{u}_{j}(\mathbf{m})-c \cdot m \geq \sum_{j \in N} \bar{u}_{j}\left(\mathbf{m}^{\prime}\right)-c \cdot m^{\prime}=W_{\mathbf{m}^{\prime}} \text { for any } \mathbf{m}, \mathbf{m}^{\prime} \in M .
$$

Note that this notion of efficiency is restricted in the sense that the allocation of the budget among agents is considered to be given by the bargaining process. An absolute notion of efficiency would specify both $\mathbf{m}$ and the allocation of the budget among agents

\footnotetext{
${ }^{4}$ Histories and strategies are specified in more detail in CRB, for the unanimity case. The adaptation to the simple-majority case is immediate.
} 
that maximizes aggregate utility. Thus, the (ex-ante) efficiency would be determined independently of any bargaining process. In our setting, as we will see, due to the nontransferability of utilities the bargaining outcome is not necessarily surplus maximizing. Hence, from an ex-ante perspective, inefficient investments are not necessarily the unique source of inefficiency. Although in this section we focus our attention to the analysis of the $r$-efficient partitions, this remark would be important when comparing the welfare generated under alternative bargaining games (either unanimous or majoritarian).

\subsection{Simple-majority bargaining}

Given a partition $\mathbf{m}=\left(m_{1}, m_{2}, m_{3}\right)$, bargaining proceeds by players alternating offers. An SSPE is characterized by a triple of (stationary) expected utilities $\left(\bar{u}_{1}, \bar{u}_{2}, \bar{u}_{3}\right) .{ }^{5}$ In this equilibrium, a proposer builds a minimal coalition: ${ }^{6}$ She proposes an allocation that maximizes her utility among all allocations that are accepted by at least one responder. When there are positive consumption externalities this does not generally mean that agents with small expected utilities are always included in the winning coalition, as compensating an investing agent with higher expected utility might be optimal because of the externalities she generates. Moreover, compensations to non-investing agents can also be made by allocating a surplus to an investing agent (which, possibly, may reject that offer). Although the characterization of the bargaining equilibria is standard, to account for these possibilities requires a simple but extensive analysis, which is presented in the Appendix. There, we characterize the (unique, no-delay and symmetric) SSPE for any $\mathbf{m}$ which, due to the symmetry of the bargaining equilibrium, only depends on the number of investing agents $m$. Next proposition summarizes the equilibrium expected utilities for any possible partition.

Proposition 1 For any $\mathbf{m} \in S$, SSPE expected utilities are given by $\bar{u}_{h}=(3 \beta+1) / 3$ if $m=3, \bar{u}_{l}=1 / 3$ if $m=0$;

$$
\bar{u}_{h}=\left\{\begin{array}{cc}
1+\beta, & \text { if } \beta>1 / 2 \\
\frac{1+\beta}{3-4 \beta}, & \text { otherwise }
\end{array} \text { and } \bar{u}_{l}=\left\{\begin{array}{cl}
\beta, & \text { if } \beta>1 / 2 \\
\frac{1-\beta}{3-4 \beta}, & \text { otherwise }
\end{array},\right.\right.
$$

when $m=1$, and $\bar{u}_{h}=\frac{(2 \beta+1)^{2}}{4 \beta+3}, \bar{u}_{l}=\frac{2 \beta+4 \beta^{2}+1}{4 \beta+3}$ for $m=2$.

Proof See Appendix A.3.

Intuitively, the size of externalities $(\beta)$ determines how attractive is to give a positive share of the budget to investing agents. Accordingly, a higher $\beta$ would increase the equilibrium expected utility of those who invested. In consequence, compensating investing agents will be more 'expensive' for a higher $\beta$, and this may induce to exclude

\footnotetext{
${ }^{5}$ We omit the dependence on $\mathbf{m}$ when no confusions arises.

${ }^{6}$ This would not be necessarily the case if the committee size was greater than 4 . See Cardona and Rubí-Barceló (2014b), where this argument is developed.
} 
them from the winning coalition (recall that this may happen under simple-majority). This will delimit the equilibrium expected utility of investing agents. ${ }^{7}$

Using this result, next two propositions characterize stable and $r$-efficient partitions. ${ }^{8}$

PROPOSITION 2 Under simple-majority bargaining, the SSPE yield stable partition $m^{*}$ given by:

1. If $\beta>3 / 4$ then
(i) $m^{*}=3$ if $c<7 \frac{\beta}{12 \beta+9}$;
(ii) $m^{*}=1$ if $c \in\left[\frac{\beta+1}{4 \beta+3}, \frac{3 \beta+2}{3}\right]$ and
(iii) $m^{*}=0$ if $c>\frac{3 \beta+2}{3}$

2. If $\beta \in[1 / 2,3 / 4]$ then
(i) $m^{*}=3$ if $c<7 \frac{\beta}{12 \beta+9}$;
(ii) $m^{*}=2$ if $c \in\left[7 \frac{\beta}{12 \beta+9}, \frac{\beta+1}{4 \beta+3}\right]$;
(iii) $m^{*}=1$ if $c \in\left[\frac{\beta+1}{4 \beta+3}, \frac{3 \beta+2}{3}\right]$ and
(iv) $m^{*}=0$ if $c>\frac{3 \beta+2}{3}$.

3. If $\beta<1 / 2$ then
(i) $m^{*}=3$ if $c<7 \frac{\beta}{12 \beta+9}$;
(ii) $m^{*}=2$ if $c \in\left[7 \frac{\beta}{12 \beta+9}, \beta \frac{7-16 \beta^{2}}{9-16 \beta^{2}}\right]$;
(iii) $m^{*}=1$ if $c \in\left[\beta \frac{7-16 \beta^{2}}{9-16 \beta^{2}}, \frac{7 \beta}{9-12 \beta}\right]$ and
(iv) $m^{*}=0$ if $c>\frac{7 \beta}{9-12 \beta}$.

PROOF Immediate from comparing the utilities of those agents who change their status given any $\mathbf{m}$ by using the bargaining equilibrium expected utilities given by Proposition 1.

\footnotetext{
${ }^{7}$ Eraslan (2002) highlights that being stronger in unanimity bargaining games does not necessarily translate into stronger positions when consensus requirements are weaker than unanimity. Along the same lines, Montero (2008) shows that altruism (an alternative interpretation of consumption externalities) may increase the agent's material payoff in a three-player majority bargaining game.

${ }^{8}$ Interestingly, for some tuples $(\beta, c)$ two equilibria are attained. If $\beta>3 / 4$ and $c \in$ $\left(\frac{\beta+1}{4 \beta+3} \frac{7 \beta}{12 \beta+9}\right)$ then both $m^{*}=1$ and $m^{*}=3$ are stable partitions.
} 
Proposition 3 The r-efficient partition $m^{0}$ is given by:

1. If $\beta>1 / 2$ then (i) $m^{0}=0$ if $c \geq 3 \beta$ and (ii) $m^{0}=1$ if $c<3 \beta$.

2. If $\beta \in[1 / 4,1 / 2]$ then (i) $m^{0}=0$ if $c \geq \frac{3 \beta}{3-4 \beta}$; (ii) $m^{0}=1$ if $c \in\left[3 \beta \frac{1-2 \beta}{3-4 \beta}, \frac{3 \beta}{3-4 \beta}\right]$ and (iii) $m^{0}=3$ if $c<3 \beta \frac{1-2 \beta}{3-4 \beta}$.

3. If $\beta<1 / 4$ then (i) $m^{0}=0$ if $c \geq \frac{3 \beta}{3-4 \beta}$; (ii) $m^{0}=1$ if $c \in\left[3 \beta \frac{3-16 \beta^{2}}{9-16 \beta^{2}}, \frac{3 \beta}{3-4 \beta}\right]$; (iii) $m^{0}=2$ if $c \in\left[3 \frac{\beta}{4 \beta+3}, 3 \beta \frac{3-16 \beta^{2}}{9-16 \beta^{2}}\right]$ and (iv) $m^{0}=3$ if $c<3 \frac{\beta}{4 \beta+3}$.

PROOF Immediate from comparing the sum of utilities for any possible $\mathbf{m}$ by using the bargaining equilibrium expected utilities given by Proposition 1 .

The comparison between SSPE and efficient investment choices given by Propositions 2 and 3 does not allow to delimit in a clear way the different combinations of stable and $r$-efficient partitions according to the parameters $c$ and $\beta$. Table 1 illustrates some pairs $(c, \beta)$ that induce the different possibilities. Symbol $\varnothing$ refers to combinations $\left(m^{0}, m^{*}\right)$ that are never attained. As shown, almost all possible combinations $\left(m^{0}, m^{*}\right)$ can be obtained for some pair of parameters $(c, \beta)$, so that SSPE investment choices may be either efficient or may display over- or under-investment. The exception is when $m^{0}=0$, which is only compatible with $m^{*}=0$.

\begin{tabular}{ccccc}
$m^{0} \backslash m^{*}$ & 0 & 1 & 2 & 3 \\
\hline \hline 0 & $(0.1,0.2)$ & $\varnothing$ & $\varnothing$ & $\varnothing$ \\
1 & $(0.1,0.1)$ & $(0.2,0.18)$ & $(0.3,0.2)$ & $(0.3,0.15)$ \\
2 & $(0.1,0.08)$ & $(0.15,0.12)$ & $(0.2,0.15)$ & $(0.25,0.1)$ \\
3 & $(0.05,0.045)$ & $(0.08,0.065)$ & $(0.1,0.07)$ & $(0.2,0.05)$ \\
\hline
\end{tabular}

Table 1

Examples of pairs $(c, \beta)$ reproducing each possible combination $\left(m^{0}, m^{*}\right)$ under simple-majority.

\subsection{Unanimity bargaining}

When unanimity is required, CRB characterize the symmetric bargaining equilibria for any $\mathbf{m}$. For the three-player case, their results are summarized next. ${ }^{9}$

Proposition 4 (CRB, 2014) Under unanimity, the SSPE expected utilities are given by $\bar{u}_{h}(\mathbf{m})=(3 \beta+1) / 3$ if $m=3, \bar{u}_{l}(\mathbf{m})=1 / 3$ if $m=0$, and

$$
\bar{u}_{h}(\mathbf{m})=\left\{\begin{array}{cl}
\frac{\beta m+1}{m} & \text { if } \beta>1 / 3 \\
\frac{\beta m+1}{3(1-\beta(3-m))} & \text { otherwise }
\end{array} \text { and } \bar{u}_{l}(\mathbf{m})=\left\{\begin{array}{cl}
\beta & \text { if } \beta>1 / 3 \\
1 / 3 & \text { otherwise }
\end{array},\right.\right.
$$

${ }^{9}$ Although we might opt to refer the reader to that paper, some of these results will be used in Section 4, so we decided to include them explicitly. 
when $m \in\{1,2\}$.

Likewise, from CRB we can also derive the stable and r-efficient partitions (now under the restriction of unanimous bargaining) as a function of $c$ and $\beta$. These results are summarized in Tables 2 and 3.

\begin{tabular}{lll}
$m^{*} \backslash \beta$ & \multicolumn{1}{c}{$(0,1 / 3)$} & \multicolumn{1}{c}{$(1 / 3,1)$} \\
\hline \hline 0 & $c>\frac{\beta}{1-2 \beta}$ & $c>\beta+\frac{2}{3}$ \\
1 & $c \in\left[\frac{\beta}{1-\beta}, \frac{\beta}{1-2 \beta}\right]$ & $c \in\left[\frac{1}{2}, \beta+\frac{2}{3}\right]$ \\
2 & $c \in\left[\beta, \frac{\beta}{1-\beta}\right]$ & $c \in\left[\frac{1}{3}, \frac{1}{2}\right]$ \\
3 & $c<\beta$ & $c \leq \frac{1}{3}$ \\
\hline
\end{tabular}

Table 2

Parameters that induce the stable partition $m^{*}$.

\begin{tabular}{lll}
$m^{0} \backslash \beta$ & \multicolumn{1}{c}{$(0,1 / 3)$} & $(1 / 3,1)$ \\
\hline \hline 0 & $c>\frac{\beta}{1-2 \beta}$ & $c>3 \beta$ \\
1 & $c \in\left[\frac{\beta(1-3 \beta)}{(1-2 \beta)(1-\beta)}, \frac{\beta}{1-2 \beta}\right]$ & $c<3 \beta$ \\
2 & $c \in\left[\frac{\beta(1-3 \beta)}{1-\beta}, \frac{\beta(1-3 \beta)}{(1-2 \beta)(1-\beta)}\right]$ & $\varnothing$ \\
3 & $c<\frac{\beta(1-3 \beta)}{1-\beta}$ & $\varnothing$ \\
\hline
\end{tabular}

Table 3

Parameters that induce the $r$-efficient partition $m^{0}$.

Comparing the different combinations of $m^{*}$ and $m^{0}$ according to all possible parameter combinations $(\beta, c)$ under unanimity is then immediate. We refer to CRB for a detailed characterization. As an illustrative example, Table 4 shows tuples of $(c, \beta)$ inducing these possibilities.

\begin{tabular}{ccccc}
$m^{0} \backslash m^{*}$ & 0 & 1 & 2 & 3 \\
\hline \hline 0 & $(0.5,0,1)$ & $\varnothing$ & $\varnothing$ & $\varnothing$ \\
1 & $(1.25,0.5)$ & $(1,0.6)$ & $(0.4,0.6)$ & $(0.2,0.6)$ \\
2 & $\varnothing$ & $\varnothing$ & $\varnothing$ & $(0.15,0.2)$ \\
3 & $\varnothing$ & $\varnothing$ & $\varnothing$ & $(0.1,0.2)$ \\
\hline
\end{tabular}

Table 4

Examples of pairs $(c, \beta)$ reproducing each possible combination $\left(m^{0}, m^{*}\right)$ under unanimity.

REMARK 1 These results are extensive to the $n$-player case where $m^{0}=1$ is consistent with any $\mathrm{m}^{*}$; thus, investments can be r-efficient or display either over- or under-investment. Otherwise, $m^{0} \in\{0, n\}=0$ is consistent only with r-efficient SPPE $\left(m^{0}=m^{*}\right)$ and any other $\operatorname{SSPE}\left(m^{*} \in\{2, \ldots, n-1\}\right)$ entails over-investment.

Worthy, from Proposition 4, it is immediate that when $\beta>1 / 3$ the bargaining outcome maximizes collective surplus only if $m \in\{1,2\}$. Hence, when $m \in\{1,2\}$ and $\beta<1 / 3$ (ex-ante) inefficiencies may arise from two different channels: An inefficient bargaining outcome and $r$-inefficient investment choices.

\section{Welfare comparisons}

Requiring unanimity at the bargaining stage makes investments particularly profitable because the increase in the expected utility obtained by investing agents when proposing 
(they can keep a bigger share of the budget) also affects positively the utility they obtain when responding (having a larger expected utility implies that they must be compensated accordingly). Contrarily, under weaker consensus requirements, proposers can exclude investing agents from the winning coalition if their demands are relatively too high. Thus, some potential benefits from investing are eroded, which reduces the incentives to invest. $^{10}$

COROLLARY 1 When decisions require simple majority the incentives to invest are lower than under unanimity.

Proof The claim follows directly from comparing the SSPE expected utilities under simple-majority (Proposition 1) and under unanimity (Proposition 4). See Appendix A.4.

This result reveals that unanimity allocates a bigger expected share of the budget to investing agents, implying that the aggregate surplus under unanimous bargaining is never smaller than under simple-majority bargaining. This is shown in Table 5, that displays total gross welfare (excluding investment costs) under unanimous and simplemajority bargaining for different values of $m$, represented by $\bar{W}_{u}(m)$ and $\bar{W}_{s m}(m)$, respectively. We use the term $\bar{W}(m)$ when $\bar{W}_{u}(m)=\bar{W}_{s m}(m)$.

\begin{tabular}{ccccccc}
$\beta$ & $\bar{W}(3)$ & $\bar{W}_{u}(2)$ & $\bar{W}_{s m}(2)$ & $\bar{W}_{u}(1)$ & $\bar{W}_{s m}(1)$ & $\bar{W}_{u}(0)$ \\
\hline \hline$>3 / 4$ & $3 \beta+1$ & $3 \beta+1$ & $\frac{12 \beta^{2}+10 \beta+3}{4 \beta+3}$ & $3 \beta+1$ & $3 \beta+1$ & 1 \\
{$[1 / 2,3 / 4]$} & $3 \beta+1$ & $3 \beta+1$ & $\frac{12 \beta^{2}+10 \beta+3}{4 \beta+3}$ & $3 \beta+1$ & $3 \beta+1$ & 1 \\
{$[1 / 3,1 / 2]$} & $3 \beta+1$ & $3 \beta+1$ & $\frac{12 \beta^{2}+10 \beta+3}{4 \beta+3}$ & $3 \beta+1$ & $\frac{3-\beta}{3-4 \beta}$ & 1 \\
$<1 / 3$ & $3 \beta+1$ & $\frac{1+\beta}{1-\beta}$ & $\frac{12 \beta^{2}+10 \beta+3}{4 \beta+3}$ & $\frac{1-\beta}{1-2 \beta}$ & $\frac{3-\beta}{3-4 \beta}$ & 1 \\
\hline
\end{tabular}

\section{Table 5}

Gross welfare for any partition under unanimity and under simple-majority.

Direct computations show that $\bar{W}_{u}(m) \geq \bar{W}_{s m}(m)$, with strict inequality when (i) $m=2$ and when (ii) $m=1$ and $\beta<1 / 2$. Nevertheless, as shown in the previous section, our model presents another source of inefficiency: stable partitions may involve either over- or under-investment, in terms of r-efficiency. Thus, although unanimity bargaining generates (weakly) more surplus for any given partition, simple majority contributes to alleviating over-investment. Hence, in order to analyze the (unrestricted) efficiency properties of the equilibria under different consensus requirements, we must study how these two opposite effects compare to each other. Then, it can be said that simple-majority will perform better than unanimity only when the over-investment reduction compensates the more inefficient bargaining allocation.

In order to illustrate the forces that interact in this comparison, we next consider two sub-cases: large externalities $(\beta>3 / 4)$ and small externalities $(\beta<1 / 6)$. A

\footnotetext{
${ }^{10}$ As noticed, this result in in line with Eraslan (2002), and contrasts with Harstad (2005).
} 
complete view of all cases is provided in Table A1 in the Appendix A.5. Table 6 displays the information contained in the first column of Table A1 consisting of the range of investment costs $(c)$ that sustain different pairs $\left(m_{s m}^{*}, m_{u}^{*}\right)$ when $\beta>3 / 4$; and also the associated net welfare gains $\Delta=\bar{W}_{u}\left(m_{u}^{*}\right)-c \cdot m_{u}^{*}-\left[\bar{W}_{s m}\left(m_{s m}^{*}\right)-c \cdot m_{s m}^{*}\right]$ of unanimity, where $m_{s m}^{*}$ and $m_{u}^{*}$ denote the stable partitions attained under simple majority and unanimity, respectively.

\begin{tabular}{cccccc}
$\left(m_{u}^{*}, m_{s m}^{*}\right)$ & $(3,3)$ & $(3,1)$ & $(2,1)$ & $(1,1)$ & $(0,0)$ \\
\hline \hline$c$ & $\left(0, \frac{7 \beta}{12 \beta+9}\right]$ & {$\left[\frac{\beta+1}{4 \beta+3}, \frac{1}{3}\right]$} & {$\left[\frac{1}{3}, \frac{1}{2}\right]$} & {$\left[\frac{1}{2}, \frac{3 \beta+2}{3}\right]$} & {$\left[\frac{3 \beta+2}{3}, \infty\right)$} \\
$\Delta$ & 0 & $-2 c$ & $-c$ & 0 & 0 \\
\hline
\end{tabular}

\section{Table 6}

Welfare comparisons between unanimity and simple-majority when $\beta>3 / 4$.

As shown, simple-majority cannot be worse than unanimity in terms of aggregate welfare. The reason lies on the fact that when externalities are large enough the equilibrium bargaining allocation is efficient under both simple-majority and unanimous bargaining (see Propositions 1 and 4). Hence, simple-majority performs better because it reduces the over-investment inefficiency. Instead, for low externalities the bargaining outcome does not maximize collective surplus. In these cases, low incentives to invest may have the opposite effect. Table 7 reproduces the same information for $\beta<1 / 6$ (in this case, $\left(m_{u}^{*}, m_{s m}^{*}\right) \in\{(3,3),(0,0)\}$ have not been included in the table for space reasons; both combinations induce the same welfare). In these cases, $\Delta>0$ unless $\beta \in(1 / 9,1 / 6)$ and $c \in\left[\frac{3 \beta(1-2 \beta)}{(3-4 \beta)}, \frac{7 \beta}{9-12 \beta}\right]$, where $\left(m_{u}^{*}, m_{s m}^{*}\right)=(3,1)$ is attained. That is, the efficiency gains at the bargaining stage under unanimity compensate the more probable inefficient (over-)investment choices almost in all cases. For intermediate values of $\beta$ there would be some variability in the dominance relationship between the alternative consensus.

\begin{tabular}{cccccc}
$\left(m_{u}^{*}, m_{s m}^{*}\right)$ & $(3,2)$ & $(3,1)$ & $(3,0)$ & $(2,0)$ & $(1,0)$ \\
\hline \hline$c$ & {$\left[\frac{7 \beta}{12 \beta+9}, \frac{\left(16 \beta^{2}-7\right) \beta}{16 \beta^{2}-9}\right]$} & {$\left[\frac{\left(16 \beta^{2}-7\right) \beta}{16 \beta^{2}-9}, \frac{7 \beta}{9-12 \beta}\right]$} & {$\left[\frac{7 \beta}{9-12 \beta}, \beta\right]$} & {$\left[\beta, \frac{\beta}{1-\beta}\right]$} & {$\left[\frac{\beta}{1-\beta}, \frac{\beta}{1-2 \beta}\right]$} \\
$\Delta$ & $-\frac{(3 c-3 \beta+4 c \beta)}{4 \beta+3}$ & $-\frac{2\left(3 \beta-3 c+4 c \beta-6 \beta^{2}\right)}{4 \beta-3}$ & $3 \beta-3 c$ & $3 \beta-2 c$ & $3 \beta-c$ \\
& $(+)$ & $(+,-)$ & $(+)$ & $(+)$ & $(+)$ \\
\hline
\end{tabular}

Table 7

Welfare comparisons between unanimity and simple-majority when $\beta<1 / 6$. 


\section{Conclusions}

We studied the situation where a set of players must decide whether to invest in generating positive consumption externalities before some budget is distributed through a simple-majority bargaining. We showed that, in this setting, the level of investment might be either efficient, too low or too high, depending on both investment costs and the size of externalities. Compared to the analogous setting with unanimity bargaining (analyzed in CRB), the over-investment inefficiency is alleviated because simple-majority generates lower incentives to invest: the acceptance of investing agents might not be needed to approve an allocation. This has a counterpart: investing agents might receive a smaller share of the budget under simple-majority bargaining, so the aggregated surplus attained at the negotiation stage might also be smaller. Hence, the comparison between the collective surpluses attained under simple-majority and unanimity bargaining is uncertain. We showed that under unanimity bargaining the equilibria are generally more efficient when the size of externalities is relatively small because in that case the over-investment inefficiency is offset by the gains from allocating a bigger share of the budget to investing agents. Contrarily, the aggregate surplus under unanimity bargaining is smaller than under simple-majority bargaining when externalities are large enough.

\section{Appendix}

\section{A.1 No-delay}

Lemma 1 Any SSPE is a no-delay equilibrium and $\sum_{i \in N} x_{i}^{j}=1$ for all $j \in N$.

Proof Consider an SSPE yielding expected utilities $\left(\bar{u}_{1}, \ldots, \bar{u}_{n}\right)$.

Because of stationarity and the linearity of preferences, the expected utility of any agent $j$ is no more than $x_{i}^{e}+\beta y^{e}$, where $x_{j}^{e}$ is the expected share obtained by agent $j$ and $y^{e}$ is the expected share allocated to investing agents at any bargaining period. This implies that, $A=\left\{\mathbf{x} \in \mathbb{R}_{+}^{n}, \sum_{i \in N} x_{j}^{i} \leq 1: u_{j}(\mathbf{x}) \geq \bar{u}_{j}\right.$ for all $\left.j \in N\right\} \neq \varnothing$. Hence, for any $\delta<1$,

$$
A(\delta)=\left\{\mathbf{x} \in \mathbb{R}_{+}^{n}, \sum x_{j}^{i}<1: u_{j}(\mathbf{x}) \geq \delta \bar{u}_{j} \text { for all } i \in N\right\} \neq \varnothing .
$$

Therefore, the proposer $j$ can obtain more than $\delta \bar{u}_{j}$ when proposing, implying that there is no delay. Moreover, $j$ will exhaust all resources when proposing so that $\sum_{i \in N} x_{i}^{j}=1$.

\section{A.2 Symmetry}

We next show that any bargaining equilibrium is symmetric. Note that this is immediate when $m=0$ or $m=3$, as agents would play the standard random proposers bargaining 
game with surplus of 1 , where a utility of $\beta$ is additionally guaranteed in the latter. Thus, we will restrict to cases where $m \in\{1,2\}$.

For any SSPE, we denote by $\bar{u}_{i}$ as the expected utility of agent $i$ and by $u_{i j}$ the expected utility obtained by responder $j$ when $i$ proposes; i.e., $u_{i j}=u_{j}\left(\mathbf{x}^{i}\right)$. Hence, the following holds:

$$
3 \bar{u}_{i}=u_{1 i}+u_{2 i}+u_{3 i} \text { for all } i=1,2,3 \text {. }
$$

Under stationary, agent $i$ would accept any proposal that gives her at least utility $\bar{u}_{i}$; i.e., any $\mathbf{x} \in A_{i}$.

LEMMA 2 If $m=1$ and an $l$-player makes offers to obtain the acceptance of the other $l$ - player then she compensates the responder through agent $h$ iff $\beta \geq 1 / 2$.

ProOF Let assume w.l.o.g. $\mathbf{m}=(1,0,0)$, and consider an equilibrium where agent 2 makes an offer in order to obtain the acceptance of 3 . This can be done using either direct compensations (i.e., $x_{3}^{2}>0$ and $x_{1}^{2}=0$ ) or compensating agent 3 through the externalities generated by 1 (i.e., $x_{3}^{2}=0$ and $x_{1}^{2}>0$ ). Using direct compensations it is required that $x_{3}^{2}=\bar{u}_{3}$, so that $u_{22}=1-x_{3}^{2}=1-\bar{u}_{3}$. Instead, using indirect compensations, $x_{1}^{2}$ must satisfy $\beta x_{1}^{2}=\bar{u}_{3}$, so that $u_{22}=1-x_{1}^{2}+\beta x_{1}^{2}=1+\bar{u}_{3}-\beta^{-1} \bar{u}_{3}$. Hence, is is immediate that 2 prefers indirect compensations iff $\beta \geq 1 / 2$.

LEMma 3 If $\mathbf{m}=(1,0,0)$ then $\bar{u}_{2}=\bar{u}_{3}$.

Proof Suppose $\bar{u}_{2}>\bar{u}_{3}$. This implies that agent 1 would never offer a positive share to the more demanding agent. I.e., $x_{2}^{1}=0$, implying $u_{12} \leq u_{13}$ (i). Regarding the equilibrium proposals of players 2 and 3 , we next consider the different possibilities, in turn.

Suppose first that 2 obtains the acceptance of player 1 . That is, $u_{1}\left(x^{2}\right)=\bar{u}_{1}$. I.e., $u_{23}=\beta x_{1}^{2}$. As $\bar{u}_{2}>\bar{u}_{3}$, this implies that it is also optimal for 3 to propose $x^{3}$ satisfying $u_{1}\left(x^{3}\right)=\bar{u}_{1}$, too. Hence, $u_{22}=u_{33}$ (ii) and $u_{23}=u_{32}$ (iii). Thus, from (i), (ii) and (iii),

$$
3 \bar{u}_{2}=u_{12}+u_{22}+u_{32} \leq u_{13}+u_{23}+u_{33}=3 \bar{u}_{3}
$$

contradicting $\bar{u}_{2}>\bar{u}_{3}$.

Suppose that 2 compensates player 3 . That is, $u_{3}\left(x^{2}\right)=u_{23}=\bar{u}_{3}$. By Lemma 2, this is done either through direct compensations $(\beta \leq 1 / 2)$ or indirectly $(\beta \geq 1 / 2)$. In the first case, $x_{3}^{2}=\bar{u}_{3}$ so that $u_{22}=1-\bar{u}_{3}$ (iv), and in the second $\beta x_{1}^{2}=\bar{u}_{3}$ so that $u_{22}=\bar{u}_{3}+1-\bar{u}_{3} / \beta$. Notice that $u_{33} \geq 1-\bar{u}_{2}$ (also when $u_{22}=1-\bar{u}_{3}$ ) because agent 3 has the option to compensate agent 2 , and when $u_{22}=\bar{u}_{3}+1-\bar{u}_{3} / \beta$, agent 3 may also obtain $u_{33}=u_{22}$ (vi) by compensating agent 1 . Notice also that $u_{32} \leq \bar{u}_{2}$ because $u_{32}>\bar{u}_{2}$ cannot be possible either if 3 compensates 2 (directly or indirectly) or if 3 compensates 1 because then player 3 would prefer to compensate 2 . Thus,

$$
3 \bar{u}_{2} \leq u_{12}+u_{22}+\bar{u}_{2}
$$




$$
3 \bar{u}_{3}=u_{13}+\bar{u}_{3}+u_{33}
$$

Then, from (i), we obtain

$$
2\left(\bar{u}_{2}-\bar{u}_{3}\right) \leq u_{22}-u_{33}
$$

Hence, from (iv) and (v), $u_{22}-u_{33} \leq \bar{u}_{2}-\bar{u}_{3}$ when $\beta<1 / 2$ and, from (vi), $u_{22}-u_{33}=0$ otherwise; this contradicts $\bar{u}_{2}>\bar{u}_{3}$.

LEMMA 4 If $\mathbf{m}=(1,1,0)$ then $\bar{u}_{1}=\bar{u}_{2}$.

Proof Suppose $\bar{u}_{1}>\bar{u}_{2}$. Then, agent 3 will never compensate agent 1 . That is, $x^{3}$ satisfies $u_{2}\left(\mathbf{x}^{3}\right)=u_{32}=(1+\beta) x_{2}^{3}=\bar{u}_{2}$ (i). Thus, $u_{31}=\frac{\beta}{1+\beta} \bar{u}_{2}<\bar{u}_{2}<\bar{u}_{1}$ (ii).

Suppose first that $x^{1}$ satisfies $u_{3}\left(x^{1}\right)=\bar{u}_{3}$ (with some probability $p$ ). Hence, as $\bar{u}_{1}>\bar{u}_{2} 2$ would also choose $x^{2}$ satisfying $u_{3}\left(x^{2}\right)=\bar{u}_{3}$, implying $u_{22}=u_{11}$ (iii) and $u_{21}=u_{12}$ (iv). Hence, from (ii) and (i),

$$
\begin{aligned}
& 3 \bar{u}_{1}<u_{11}+u_{21}+\bar{u}_{1} \\
& 3 \bar{u}_{2}=u_{12}+u_{22}+\bar{u}_{2} .
\end{aligned}
$$

Then, from (iii) and (iv), $2\left(u_{1}-u_{2}\right)<0$, contradicting $\bar{u}_{1}>\bar{u}_{2}$.

In case that 1 compensates player $2, x^{1}$ satisfies $u_{2}\left(x^{1}\right)=u_{12}=\beta x_{1}^{1}+(1+\beta) x_{2}^{1}=\bar{u}_{2}$ $(v)$. Hence, $u_{11}=\left(1+2 \beta-\bar{u}_{2}\right)$ (vi). Following similar steps, we can conclude that since agent 2 can always compensate $1, u_{21}=(1+\beta) x_{1}^{2}+\beta\left(1-x_{1}^{2}\right) \leq \bar{u}_{1}$ (vii). In consequence, $u_{22}=\beta x_{1}^{2}+(1+\beta)\left(1-x_{1}^{2}\right) \geq 1+2 \beta-\bar{u}_{1}$ (viii). Thus,

From (ii), (vi) and (vii), $3 \bar{u}_{1}<\left(1+2 \beta-\bar{u}_{2}\right)+\bar{u}_{1}+\bar{u}_{1}$, and from (i), (v) and (viii), $3 \bar{u}_{2} \geq \bar{u}_{2}+\left(1+2 \beta-\bar{u}_{1}\right)+\bar{u}_{2}$

From these two inequalities, we obtain that $\bar{u}_{1}-\bar{u}_{2}<\bar{u}_{1}-\bar{u}_{2}$, i.e. a contradiction. Q.E.D.

\section{A.3 Proof of Proposition 1}

In case that all agents are identical, they obtain the same expected utilities. I.e., $u_{i}(\mathbf{m})=$ $1 / 3$ if $m=0$ and $u_{i}(\mathbf{m})=(1+3 \beta) / 3$ if $m=3$. Next, the other possible partitions are considered.

Using symmetry, the no-delay property of SSPE and the linearity of utilities, we can next specify the relationship between the equilibrium expected utilities of the agents when $m \in\{1,2\}$.

LEMMA 5 In any bargaining equilibrium

$$
\begin{aligned}
& \bar{u}_{h}=\frac{(1+2 \beta)}{2(1-\beta)}\left(1-\bar{u}_{l}\right) \text { when } m=2 \text { and } \\
& \bar{u}_{h}=\frac{(\beta+1)\left(2 \bar{u}_{l}-1\right)}{2 \beta-1} \text { when } m=1 \text { and } \beta \neq 1 / 2 .
\end{aligned}
$$

If $m=1$ and $\beta=1 / 2, \bar{u}_{l}=1 / 2$ and $\bar{u}_{h}=1+\beta$. 
Proof Symmetry implies expected shares of $(1-2 y, y, y)$ when $\mathbf{m}=(1,0,0)$ and $(y, y, 1-2 y)$ when $\mathbf{m}=(1,1,0)$ for some $y \in[0,1 / 2]$. This implies that when $\mathbf{m}=$ $(1,0,0), \bar{u}_{h}=(1+\beta)(1-2 y)$ and $\bar{u}_{l}=y+\beta(1-2 y)$. Rearranging terms, the result follows. The utilities for the case $\mathbf{m}=(1,1,0)$ can be obtained similarly. When $\beta=1 / 2$ and $m=1, \bar{u}_{l}=1 / 2$. So, agent 1 may obtain $u_{h}\left(x^{1}\right)=1+\beta$ when proposing because $l$-agents would accept the proposal. Since there is no discounting, agent 1 only accepts $(1,0,0)$ and $l$-agents only accept those proposals in which they get $\beta$. Thus, the result follows.

Q.E.D.

Next, we derive the bargaining expected utilities through two lemmata.

LEMMA 6 If $m=1$ expected utilities are given by

1. $\bar{u}_{h}=\frac{1+\beta}{3-4 \beta}$ and $\bar{u}_{l}=\frac{1-\beta}{3-4 \beta}$ when $\beta<1 / 2$, and

2. $\bar{u}_{h}=1+\beta$ and $\bar{u}_{l}=\beta$ otherwise.

Proof Let assume, w.l.o.g. $\mathbf{m}=(1,0,0)$. Suppose first that $\beta \geq 1 / 2$. Due to symmetry, $\bar{u}_{l}=y+\beta(1-2 y)$ for some $y \in[0,1 / 2]$. So, $l-t y p e$ agents may obtain at most $\bar{u}_{l}=\beta$. Hence, agent 1 may obtain $u_{h}\left(\mathbf{x}^{1}\right)=1+\beta$ when proposing, so that $u_{l}\left(x^{1}\right)=\beta$. Since there is no discounting, this implies that 1 accepts only $(1,0,0)$ and $l$-players accept only proposals giving them at least $\beta$. Thus, equilibrium proposals are $x^{l}=x^{h}=(1,0,0)$, proving claim 2 .

When $\beta<1 / 2$, from Lemma 2 we know that compensations among $l$-players are always made directly. Consider three alternative proposals of agent 2: She proposes $x^{2}$ satisfying $u_{3}\left(x^{2}\right)=\bar{u}_{3}, u_{1}\left(x^{2}\right)=\bar{u}_{1}$ or a mixed between them. Agent 3 acts symmetrically.

If $u_{3}\left(x^{2}\right)=\bar{u}_{3}$ then $u_{23}=x_{3}^{2}=\bar{u}_{3}$ implying $u_{22}=1-x_{3}^{2}=1-\bar{u}_{3}$. Instead, if $u_{1}\left(x^{2}\right)=$ $\bar{u}_{1}$ then $u_{21}=(1+\beta) x_{1}^{2}=\bar{u}_{1}$ implying $u_{22}=1-x_{1}^{2}+\beta x_{1}^{2}=1-(1-\beta) \bar{u}_{1} /(1+\beta)$ and $u_{23}=\beta \bar{u}_{1} /(1+\beta)$

By symmetry $\bar{u}_{2}=\bar{u}_{3}=\bar{u}_{l}$. Suppose first that agent 2 prefers to compensate agent 3 rather than 1 , i.e.

$$
1-\bar{u}_{l}>1-\frac{1-\beta}{1+\beta} \bar{u}_{1} \Leftrightarrow \bar{u}_{l}<\frac{1-\beta}{1+\beta} \bar{u}_{1}
$$

Then, $x^{2}$ satisfying $u_{3}\left(x^{2}\right)=\bar{u}_{l}$ is optimal. Hence, if $\bar{u}_{l} \leq \beta$ so that $x_{1}^{1}=1$ we obtain (as $\bar{u}_{2}=\bar{u}_{3}$ ),

$$
\begin{aligned}
3 \bar{u}_{l} & =\beta+1-\bar{u}_{l}+\bar{u}_{l} \\
3 \bar{u}_{1} & =1+\beta
\end{aligned}
$$

which contradicts (2). Instead, if $\bar{u}_{l}>\beta$, then $x_{1}^{1}<1$ and $x_{l}^{1}>0$ for some $l$ such that $\beta x_{1}^{1}+\left(1-x_{1}^{1}\right)=\bar{u}_{l}$. Therefore, by symmetry

$$
3 \bar{u}_{l}=\frac{1}{2}\left(\bar{u}_{l}+\frac{\beta}{1-\beta}\left(1-\bar{u}_{l}\right)\right)+1-\bar{u}_{l}+\bar{u}_{l} \Leftrightarrow \bar{u}_{l}=\frac{2-\beta}{5-4 \beta}
$$




$$
3 \bar{u}_{1} \leq 1+\beta
$$

Thus,

$$
\bar{u}_{l}-\frac{1-\beta}{1+\beta} \bar{u}_{h} \geq \frac{2-\beta}{5-4 \beta}-\frac{1-\beta}{3}>0
$$

contradicting (2) again. So, when $\beta<1 / 2$, a low type agent cannot compensate the other.

Suppose now that $\bar{u}_{l}>\frac{1-\beta}{1+\beta} \bar{u}_{h}$, so that a low-type agent prefers to compensate the high-type agent. Note that this is inconsistent with $\bar{u}_{l} \leq \beta$, since otherwise $\bar{u}_{1}=u_{11}=$ $1+\beta$; contradicting $\bar{u}_{l}>\frac{1-\beta}{1+\beta} \bar{u}_{1}=1-\beta$, because $\beta<1 / 2$. Thus, $\bar{u}_{l}>\frac{1-\beta}{1+\beta} \bar{u}_{1}$ is possible only if $\bar{u}_{l}>\beta$. In these cases, $x_{1}^{1}=1-x_{l}^{1}$ where $\beta x_{1}^{1}+1-x_{1}^{1}=\bar{u}_{l}$; i.e., $x_{1}^{1}=\left(1-\bar{u}_{l}\right) /(1-\beta)$, so that $u_{11}=(1+\beta)\left(1-\bar{u}_{l}\right) /(1-\beta)$. Hence,

$$
3 \bar{u}_{1}=(1+\beta) \frac{1-\bar{u}_{l}}{1-\beta}+\bar{u}_{1}+\bar{u}_{1}
$$

yielding $\bar{u}_{1}=(1+\beta) \frac{1-\bar{u}_{l}}{1-\beta}$. Therefore,

$$
\bar{u}_{l}>\frac{1-\beta}{1+\beta} \bar{u}_{1}=1-\bar{u}_{l} \Leftrightarrow \bar{u}_{l}>1 / 2
$$

However, as $\beta<1 / 2$ this is never possible.

Thus, when $\beta<1 / 2$ it must be that $\bar{u}_{l}=\frac{1-\beta}{1+\beta} \bar{u}_{1}$. Using Lemma 5 , we obtain

$$
\bar{u}_{l}=\frac{1-\beta}{3-4 \beta} \text { and } \bar{u}_{h}=\frac{1+\beta}{3-4 \beta}
$$

LEMMA 7 If $m=2$ then $\bar{u}_{h}=\frac{(2 \beta+1)^{2}}{4 \beta+3}$ and $\bar{u}_{l}=\frac{2 \beta+4 \beta^{2}+1}{4 \beta+3}$.

Proof Assume w.l.o.g. that $\mathbf{m}=(1,1,0)$. Notice first that by proposing $x_{3}^{3}=0$ (which would be accepted by $h$ ) and accepting any proposal agent 3 may obtain $\beta$. Thus, $\bar{u}_{l} \geq \beta$. Moreover, it is immediate that $x_{3}^{3}=1$ cannot be sustained in equilibrium, since this would imply $\bar{u}_{h}=0$. So, in any equilibrium $x^{3}$ satisfies

$$
\left(1-x_{3}^{3}\right)(1+\beta)=\bar{u}_{h} \Longleftrightarrow 1-x_{3}^{3}=\frac{1}{1+\beta} \bar{u}_{h},
$$

implying

$$
\begin{aligned}
& u_{31}=p \bar{u}_{h}+(1-p) \frac{\beta}{1+\beta} \bar{u}_{h}=\frac{1}{1+\beta}(p+\beta) \bar{u}_{h} \\
& u_{32}=(1-p) \bar{u}_{h}+p \frac{\beta}{1+\beta} \bar{u}_{h}=\frac{1}{1+\beta}(1-p+\beta) \bar{u}_{h},
\end{aligned}
$$


for some $p \in[0,1]$.

Regarding $x^{h}$, suppose first that $x_{h}^{h}=1$, which is possible if either $\bar{u}_{h} \leq \beta$ or $\bar{u}_{3} \leq \beta$. In these cases, $u_{11}=u_{22}=1+\beta$ and $u_{21}=u_{12}=\beta$ so that (due to symmetry) $p$ must be $1 / 2$. Hence,

$$
3 \bar{u}_{h}=1+2 \beta+\frac{1}{1+\beta}\left(\frac{1}{2}+\beta\right) \bar{u}_{h} \Rightarrow \bar{u}_{h}=\frac{1}{4 \beta+5}\left(6 \beta+4 \beta^{2}+2\right) \in(\beta, 1+\beta) .
$$

Thus,

$$
\begin{aligned}
3 \bar{u}_{3} & =2 \beta+x_{3}^{3}+\beta\left(1-x_{3}^{3}\right)=2 \beta+\left(1-\frac{\bar{u}_{h}}{1+\beta}\right)+\beta \frac{\bar{u}_{h}}{1+\beta} \\
& =2 \beta+1-\frac{(1-\beta) \bar{u}_{h}}{1+\beta},
\end{aligned}
$$

so that $\bar{u}_{3}>\beta$, which is a contradiction. This also implies that $\bar{u}_{h}>\beta$.

Thus, in any equilibrium $h$-type agents cannot obtain all surplus. These agents must choose between compensating agent 3 or the other $h$-type: By choosing $x^{1}$ satisfying $u_{3}\left(x^{1}\right)=\bar{u}_{3}$ and therefore $\beta x_{1}^{1}+\left(1-x_{1}^{1}\right)=\bar{u}_{3}$ player 1 can obtain $u_{11}=\frac{1+\beta}{1-\beta}\left(1-\bar{u}_{3}\right)$; and by choosing $x^{1}$ satisfying $u_{2}\left(x^{1}\right)=\bar{u}_{2}$ and therefore $\beta x_{1}^{1}+(1+\beta)\left(1-x_{1}^{1}\right)=\bar{u}_{2}$ he can obtain $u_{11}=2 \beta+1-\bar{u}_{2}$ (The symmetric argument applies to player 2 ).

In case that

$$
\frac{1+\beta}{1-\beta}\left(1-\bar{u}_{3}\right)>2 \beta+1-\bar{u}_{2}
$$

then it is optimal for $h$-players to compensate agent 3 . Thus,

$$
3 \bar{u}_{3}=2 \bar{u}_{3}+\left(1-\frac{\bar{u}_{h}}{1+\beta}\right)+\beta \frac{\bar{u}_{h}}{1+\beta} \Rightarrow \bar{u}_{3}=1-\frac{(1-\beta)}{1+\beta} \bar{u}_{h}
$$

contradicting Lemma 5.

Suppose now that

$$
\frac{1+\beta}{1-\beta}\left(1-\bar{u}_{3}\right)<2 \beta+1-\bar{u}_{2}
$$

in which case, it is optimal for $h$-players to compensate the other $h$ - agent. Thus, using symmetry,

$$
\begin{aligned}
3 \bar{u}_{h} & =\left(2 \beta+1-\bar{u}_{h}\right)+\bar{u}_{h}+\frac{1}{2}\left(\bar{u}_{h}+\frac{\beta}{1+\beta} \bar{u}_{h}\right) \Rightarrow \bar{u}_{h}=\frac{1}{4 \beta+5}\left(6 \beta+4 \beta^{2}+2\right) \\
3 \bar{u}_{3} & =2 \beta+1-\frac{(1-\beta)}{1+\beta} \bar{u}_{h} \Rightarrow \bar{u}_{3}=\frac{(1+2 \beta)^{2}}{4 \beta+5},
\end{aligned}
$$

contradicting (3). 
Thus, in any equilibrium we must have that

$$
\frac{1+\beta}{1-\beta}\left(1-\bar{u}_{3}\right)=2 \beta+1-\bar{u}_{2}
$$

which, using Lemma 5 yields,

$$
\bar{u}_{h}=\frac{(2 \beta+1)^{2}}{4 \beta+3} \text { and } \bar{u}_{l}=\frac{2 \beta+4 \beta^{2}+1}{4 \beta+3} .
$$

It is not difficult to show that such an equilibrium is sustained when $h$-type agents compensate the $l-$ type agent with probability $p=\frac{1}{2(1+\beta)}$.

Q.E.D.

\section{A.4 Proof of Corollary 1}

Using Propositions 1 and 4, we can compare the expected utilities of the agents for any partition of the committee members; thus the profitability of investment. The results are summarized next. The terms $m \rightarrow m+1$ refer to the individual profitability of investing from an initial partition with $m$ investing agents. Such a profitability might depend on the exact value of $\beta$, specified into brackets when it applies.

$$
\begin{array}{lll}
m \rightarrow m+1 & \text { Simple majority } & \text { Unanimity } \\
\hline \hline & & \\
0 \rightarrow 1 & \frac{7 \beta}{9-12 \beta}[\beta \leq 1 / 2], 2 / 3+\beta[\beta>1 / 2] & \frac{\beta}{1-2 \beta}[\beta \leq 1 / 3], 2 / 3+\beta[\beta>1 / 3] \\
1 \rightarrow 2 & \beta \frac{16 \beta^{2}-7}{16 \beta^{2}-9}[\beta \leq 1 / 2], \frac{\beta+1}{4 \beta+3}[\beta>1 / 2] & \frac{\beta}{1-\beta}[\beta \leq 1 / 3], \frac{1}{2}[\beta>1 / 3] \\
2 \rightarrow 3 & \frac{\beta}{12 \beta+9} & \beta[\beta \leq 1 / 3], \frac{1}{3}[\beta>1 / 3]
\end{array}
$$

Comparing profitability it is immediate that in any case, and for any value of $\beta$ the term in the unanimity column is always greater than that in the simple majority column.

\section{A.5 Stable partitions}

Using Table 2 and Proposition 2, and after tedious comparisons, next table displays the stable partitions under unanimous bargaining $\left(m_{u}^{*}\right)$ and simple-majority bargaining $\left(m_{s m}^{*}\right)$, that are obtained for different values of $(\beta, c)$. Intervals in the table refer to values of $c$ for which $\left(m_{u}^{*}, m_{s m}^{*}\right)$ are attained, and symbol $\varnothing$ refers to situations where $\left(m_{u}^{*}, m_{s m}^{*}\right)$ can never be attained. 


\begin{tabular}{|c|c|c|c|c|c|c|}
\hline$\left(m_{u}^{*}, m_{s m}^{*}\right)$ & $\beta>3 / 4$ & $\beta \in[1 / 2,3 / 4]$ & $\beta \in[0.34615,1 / 2]$ & $\beta \in[1 / 3,0.34615]$ & $\beta \in[1 / 3,1 / 6]$ & $\beta<1 / 6$ \\
\hline$(3,3)$ & $\overline{\left(0, \frac{7 \beta}{12 \beta+9}\right.}$. & $\left(0, \frac{7 \beta}{12 \beta+9}\right]$ & $\left(0, \frac{7 \beta}{12 \beta+9}\right)$ & $\left(0, \frac{7 \beta}{12 \beta+9}\right)$ & $\left(0, \frac{7 \beta}{12 \beta+9}\right)$ & $\left(0, \frac{7 \beta}{12 \beta+9}\right)$ \\
\hline$(3,2)$ & $\varnothing$ & {$\left[\frac{7 \beta}{12 \beta+9}, \frac{\beta+1}{4 \beta+3}\right]$} & $\frac{7 \beta}{12 \beta+9}, \frac{\left(16 \beta^{2}-7\right) \beta}{16 \beta^{2}-9}$ & {$\left[\frac{7 \beta}{12 \beta+9}, \frac{\left(16 \beta^{2}-7\right) \beta}{16 \beta^{2}-9}\right]$} & $\left.\frac{7 \beta}{12 \beta+9}, \frac{\left(16 \beta^{2}-7\right) \beta}{16 \beta^{2}-9}\right]$ & $\frac{7 \beta}{12 \beta+9}, \frac{\left(16 \beta^{2}-7\right) \beta}{16 \beta^{2}-9}$ \\
\hline$(3,1)$ & {$\left[\frac{\beta+1}{4 \beta+3}, \frac{1}{3}\right]$} & {$\left[\frac{\beta+1}{4 \beta+3}, \frac{1}{3}\right]$} & {$\left[\frac{\left(16 \beta^{2}-7\right) \beta}{16 \beta^{2}-9}, \frac{1}{3}\right]$} & {$\left[\frac{\left(16 \beta^{2}-7\right) \beta}{16 \beta^{2}-9}, \frac{1}{3}\right]$} & {$\left[\frac{\left(16 \beta^{2}-7\right) \beta}{16 \beta^{2}-9}, \beta\right]$} & {$\left[\frac{\left(16 \beta^{2}-7\right) \beta}{16 \beta^{2}-9}, \frac{7 \beta}{9-12 \beta}\right.$} \\
\hline$(3,0)$ & $\varnothing$ & $\varnothing$ & $\varnothing$ & $\varnothing$ & $\varnothing$ & {$\left[\frac{7 \beta}{9-12 \beta}, \beta\right]$} \\
\hline$(2,2)$ & $\varnothing$ & $\varnothing$ & $\varnothing$ & $\varnothing$ & $\varnothing$ & {$\left[\begin{array}{l}{[9-12 \beta} \\
\varnothing\end{array}\right]$} \\
\hline$(2,1)$ & {$\left[\frac{1}{3}, \frac{1}{2}\right]$} & {$\left[\frac{1}{3}, \frac{1}{2}\right]$} & {$\left[\frac{1}{3}, \frac{1}{2}\right]$} & {$\left[\frac{1}{3}, \frac{7 \beta}{9-12 \beta}\right.$} & {$\left[\beta, \frac{7 \beta}{9-12 \beta}\right]$} & $\varnothing$ \\
\hline$(2,0)$ & $\varnothing$ & $\varnothing$ & $\varnothing$ & {$\left[\frac{7 \beta}{9-12 \beta}, \frac{1}{2}\right]$} & {$\left[\frac{7 \beta}{9-12 \beta}, \frac{\beta}{1-\beta}\right]$} & {$\left[\beta, \frac{\beta}{1-\beta}\right]$} \\
\hline$(1,1)$ & {$\left[\frac{1}{2}, \frac{3 \beta+2}{3}\right]$} & {$\left[\frac{1}{2}, \frac{3 \beta+2}{3}\right]$} & {$\left[\frac{1}{2}, \frac{7 \beta}{9-12 \beta}\right]$} & $\varnothing$ & $\varnothing$ & $\varnothing$ \\
\hline$(1,0)$ & $\varnothing$ & $\varnothing$ & {$\left[\frac{7 \beta}{9-12 \beta}, \frac{3 \beta+2}{3}\right]$} & {$\left[\frac{1}{2}, \frac{3 \beta+2}{3}\right]$} & {$\left[\frac{\beta}{1-\beta}, \frac{\beta}{1-2 \beta}\right.$} & {$\left[\frac{\beta}{1-\beta}, \frac{\beta}{1-2 \beta}\right]$} \\
\hline$(0,0)$ & {$\left[\frac{3 \beta+2}{3}, \infty\right)$} & {$\left[\frac{3 \beta+2}{3}, \infty\right)$} & {$\left[\frac{3 \beta+2}{3}, \infty\right)$} & {$\left[\frac{3 \beta+2}{3}, \infty\right)$} & {$\left[\frac{\beta}{1-2 \beta}, \infty\right)$} & {$\left[\frac{\beta}{1-2 \beta}, \infty\right)$} \\
\hline
\end{tabular}

Table A1

Ranges of $c$ that sustain any possible pair of stable partitions $\left(m_{u}^{*}, m_{s m}^{*}\right)$ for any possible value of $\beta$.

\section{References}

Baron, D.P., Ferejohn, J.A. (1989) "Bargaining in Legislatures" American Political Science Review, 83, 1181-1206.

Calvert R.L. and Dietz, N. (2005) "Legislative Coalitions in a Bargaining Model with Externalities" in Social Choice and Strategic Decisions, Springer Berlin Heidelberg.

Cardona, D. and Rubí-Barceló, A. (2014a) "Investments and bargaining in a model with positive consumption externalities" Journal of Economic Behavior ES Organization, 108, 78-93.

Cardona, D. and Rubí-Barceló, A. (2014b) "On the efficiency of equilibria un a legislative bargaining model with particularistic and collective goods" Public Choice, 161, 345-366.

Che, Y-K. and Hausch D. (1999) "Cooperative Investments and the Value of Contracting" American Economic Review, 89, 125-147.

de Meza, D. and Lockwood, B. (2010) "Too much investment?: a problem of endogenous outside options" Games and Economic Behavior, 69(2), 503-511.

Eraslan, H. (2002) "Uniqueness of stationary equilibrium payoff in the Baron-Ferejohn model" Journal of Economic Theory, 103, 11-30.

Grout, P. (1984) "Investment and Wages in the Absence of Binding Contracts: A Nash Bargaining Approach" Econometrica, 52, 449-460.

Harstad, (2005) "Majority Rules and Incentives" The Quarterly Journal of Economics, 15351568.

Hart, O. and Moore J. (1988) "Incomplete Contracts and Renegotiation" Econometrica, 56, 755-785.

Montero, M. (2008) "Altruism, Spite and Competition in Bargaining Games" Theory and Decision, 65(2), 125-151

Rogerson, W. P. (1992) "Contractual Solutions to the Hold-Up Problem" Review of Economic Studies, 59, 777-793. 
Daniel Cardona

Departament d'Economia Aplicada

Universitat de les Illes Balears

Edifici Jovellanos, Campus UIB, 07122

Palma de Mallorca, Illes Balears

Spain

d.cardona@uib.cat
Antoni Rubí-Barceló

Departament d'Economia Aplicada Universitat de les Illes Balears

Edifici Jovellanos, Campus UIB, 07122

Palma de Mallorca, Illes Balears

Spain

antoni.rubi@uib.eu 